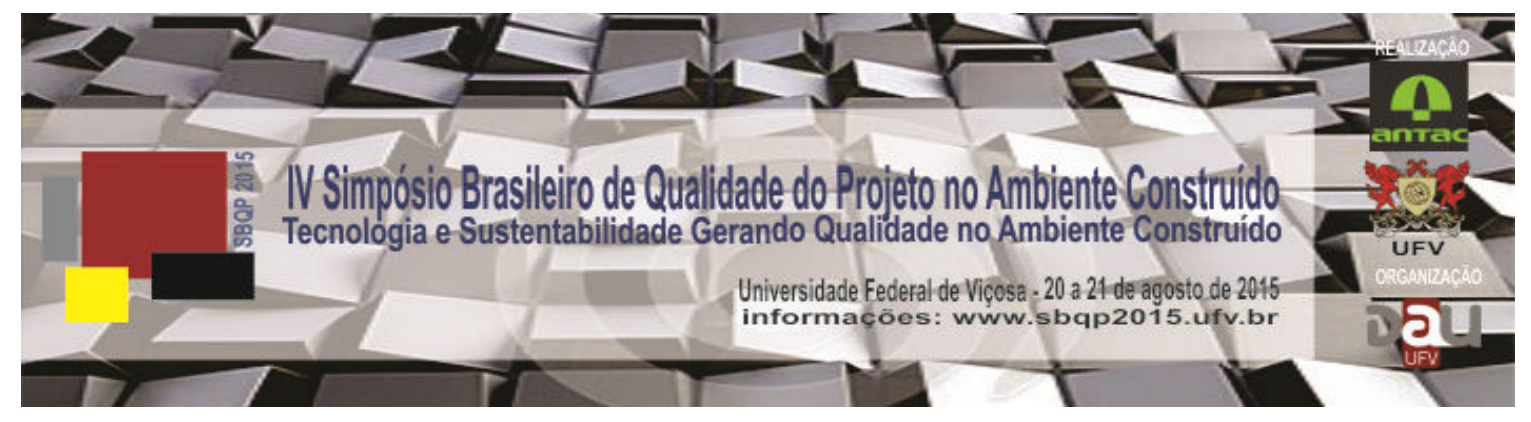

\title{
ELEMENTOS DETERMINANTES NO DESENVOLVIMENTO DE PROJETOS DE UNIDADES ASSISTENCIAIS DE SAÚDE1
}

\author{
BARBOSA, VINÍCIUS L. \\ PROARQ/FAU-UFRJ, viniciusbarbosa.arq@gmail.com
}

\begin{abstract}
RESUMO
Os projetos de arquitetura para espaços de saúde sofrem influência de diversas especialidades que estão muitas vezes aquém da formação do arquiteto. Essa interdisciplinaridade deve ser explorada no desenvolvimento do projeto, visando obter resultados que atendam às necessidades dos usuários, bem como às especificações técnicas para a articulação no espaço. Através de um levantamento bibliográfico abrangente, discussões acadêmicas no curso de Especialização em Arquitetura de Ambientes de Saúde da UFRJ e prática projetual adquirida pelos profissionais da área, foi possível identificar a ocorrência dos elementos determinantes no processo de projeto para espaços de saúde. Dessa forma verificou-se: a tecnologia, a legislação pertinente e os procedimentos médicos, como elementos condicionantes para a eficácia do resultado do projeto.
\end{abstract}

Palavras-chave: Projetos de arquitetura, Espaços de saúde, Processo de Projeto.

\begin{abstract}
The architecture projects for healthcare spaces are influenced by many specialties that are often beyond of architectural education. This interdisciplinarity should be explored in project development, in order to obtain results that meet the needs of users as well as the technical specifications for the joint space. Through a comprehensive literature review, academic discussions in the course of Specialization in Health Environments Architecture (UFRJ), and design practice acquired by professionals in the area, it was possible to identify the occurrence of the key elements in the design process for health spaces. By this was possible to find: the technology, the relevant legislation and medical procedures, as conditioning elements for the effectiveness of the outcome of the project.
\end{abstract}

Keywords: Architecture projects, Health Spaces, Design Process.

\section{INTRODUÇÃO}

A arquitetura da saúde seguiu um ritmo evolutivo intenso absorvendo e respondendo ao desenvolvimento da medicina, passando de instituição religiosa assistencialista, como nos templos greco-romanos, para instituição privada intervencionista, ilustrada pelos hospitais monoblocos saturados de

\footnotetext{
${ }^{1}$ Trabalho apresentado no IV SBQP 2015. Universidade Federal de Viçosa. Disponível em: doi>' http://dx.doi.org/10.18540/2176-4549.6037
} 
espaços técnicos, complicada infraestrutura e manutenção. Hoje o panorama é complexo, pesquisas demostram que o conceito de saúde está mudando e a atuação profissional também.

Resultado do desenvolvimento natural da profissão, o campo de trabalho do arquiteto foi fragmentado. Esse profissional que atua em projetos para instituições de saúde apresenta a necessidade de resgatar o que foi perdido e adquirir novos conhecimentos, inclusive fora do seu campo de trabalho. Segundo o engenheiro Karman (2008, p.31), "[...] tudo se interliga em um hospital, tudo se entrosa e interdepende." Ser interdisciplinar é essencial, o gerenciamento das escolhas prevendo suas consequências é uma técnica que requer amplo conhecimento das áreas envolvidas.

Por compreender a área tecnológica a mutação do espaço da saúde não terá fim. Aliadas a tecnologia, os procedimentos médicos e a legislação sanitária interagem dialogando e tomando decisões que influenciam nos fluxos, na compartimentação, no layout e na arquitetura dos ambientes de saúde, definindo o espaço arquitetônico da saúde.

Assim, com o domínio e a aplicação desses três elementos condicionantes os planejadores de saúde podem desenvolver uma perspectiva ampla e sistematizada do projeto resultando em uma arquitetura de qualidade atendendo as necessidades dos usuários do espaço.

O trabalho propõe demonstrar tal interdisciplinaridade aplicada ao processo. Discorre no campo do projeto analisando e relacionando os elementos que influenciam diretamente o programa arquitetônico dos ambientes de saúde. Desenvolver a perspectiva crítica para esse tipo de projeto é de extrema relevância para o futuro do mercado e esse pensamento é promovido em instituições de ensino e pesquisa como a Proarq na UFRJ, contribuindo também com a produção acadêmica para o segmento.

\section{MÉTODO}

Delimitando o estudo a nível preliminar foi necessário um levantamento geral das principais perspectivas técnico-científicas acerca dos assuntos em questão visando a articulação delas no espaço arquitetônico para posterior confrontamento com as informações adquiridas no campo projetual, traçando uma visão sistematizada da concepção dos espaços de saúde.

A metodologia aplicada foi a análise bibliográfica dos temas, assim como os debates acadêmicos realizados no curso de Especialização em Ambientes da Saúde da UFRJ, confluindo em uma perspectiva contemporânea acerca do assunto.

Compreender o método do projeto com suas singularidades e dominar essa técnica contribui para a excelência da arquitetura e a competitividade de qualidade no mercado profissional.

\section{TECNOLOGIA}

A tecnologia sempre influenciou o comportamento e os ambientes impactando o mercado consumidor. No campo da saúde essa atuação ocorreu de forma considerável. Medicina Nuclear, Imagenologia, Engenharia Clínica, Robotização, Telemedicina são terminologias que há muito tempo 
fazem parte do repertorio dos administradores, planejadores e profissionais de saúde. O parque tecnológico e os espaços técnicos, antes não necessários, agora fazem parte do programa de unidades de saúde, como as áreas de T.I., Conjunto Geradores, Subestações, Ar Condicionado, áreas para infraestrutura predial e manutenção, incluindo os equipamentos médicos.

Segundo Machry (2012, p. 82), "Nas áreas de diagnóstico e tratamento, que agregam equipamentos variados e complexos, a arquitetura tem enfrentado desafios crescentes para acompanhar a rapidez com que esses equipamentos têm sido criados e alterados pela indústria."

A tecnologia afeta diretamente o espaço e isso pode ser facilmente visualizado na comparação dos espaços necessários para a instalação de equipamento de radiografia nos últimos anos. Outro exemplo é a área de uma sala híbrida (figura 1), que integra a qualidade da imagem de alta definição e procedimentos cirúrgicos complexos fazendo uso de tecnologia avançada para intervenções menores e precisas, esse ambiente pode exceder em três vezes a área de uma sala cirúrgica comum não sendo prevista ainda a normatização.

A interface da tecnologia com a legislação é relevante, pois a normativa não se atualiza na velocidade do desenvolvimento tecnológico. Alguns órgãos especializados avaliam o projeto, a citar a CNEN - Comissão Nacional de Energia Nuclear que também auxilia a instalação de equipamentos radiológicos.

Outro fato relevante a ser considerado é o "ajuste de última hora" ou as "medidas temporárias" tão usuais e que tanto interferem no uso do espaço. Um equipamento instalado sem planejamento sem um estudo de impacto é prejuízo para a instituição, para alguns procedimentos e em certos casos pode até ser contra as normas. A exemplo disso podemos citar os impactos das instalações de ar-condicionado que necessitam de acústica, drenagem e localização bem definidas. 
Figura 1 - Sala Híbrida

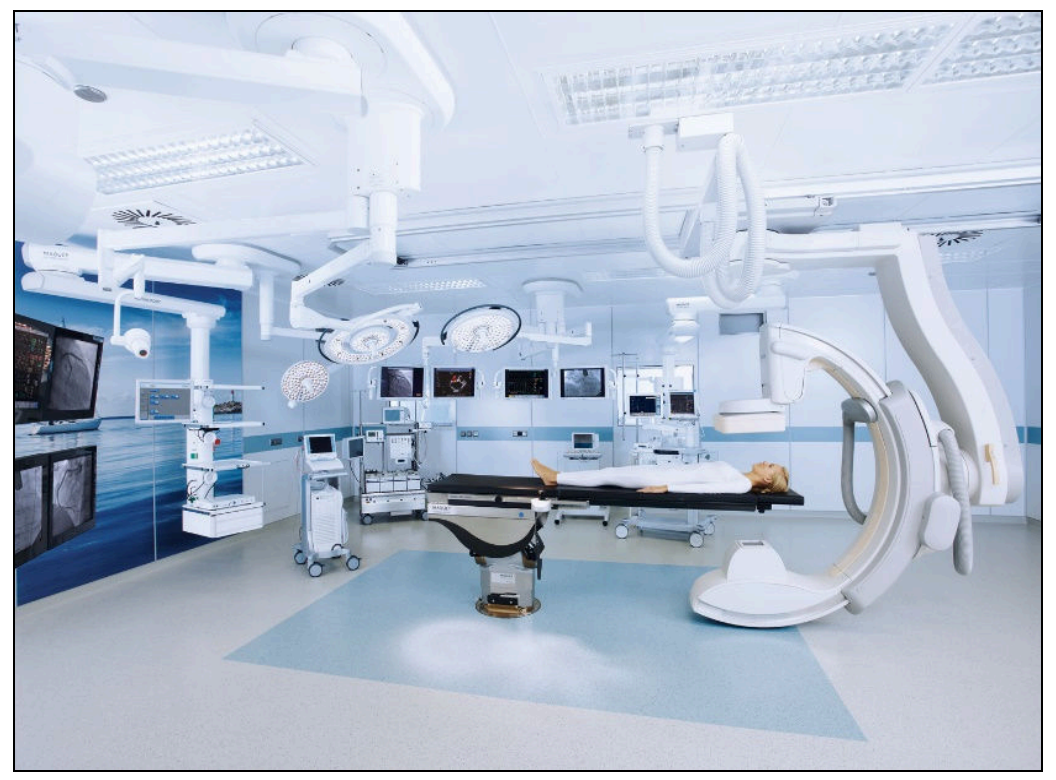

Fonte: http://www.revistahospitaisbrasil.com.br/noticias/equipamentos-hospital-israelitaalbert-einstein-tem-a-primeira-sala-hibrida-robotica-do-futuro. Acesso em: 30 mar. 2015 Sala híbrida, aumento de área e tecnologia integrada.

\section{LEGISLAÇÃO PERTINENTE}

A estrutura espacial e o dimensionamento do espaço da saúde são temas que requerem atenção nos EAS pois afetam incisivamente os usuários e disciplinalos é ainda mais delicado. Ao longo da evolução da arquitetura e dos projetos a normatização se fez necessária surgindo com a ideia de sistematizar e replicar os espaços de saúde visando melhorar o desempenho do profissional no espaço de trabalho e, atualmente, auxiliando o paciente na percepção e utilização do ambiente (figura 2).

Até o século XVIII o hospital não tinha um programa bem definido, Tenon estudou o percurso, o movimento, os ambientes, traçando fluxos resultados das práticas e procedimentos médicos que conceberam um programa, um embrião da normativa. No século XIX, surgiu o conceito de higiene pública que derivou da noção de salubridade desenvolvida no século anterior. Tal conceito relacionado com ao pensamento, então recente, de direitos humanos norteou os rumos da arquitetura hospitalar e da política mundial.

Hoje no Brasil é dever do Estado regulamentar os espaços de saúde, cabe a ele proteger e promover a saúde, que pela Constituição de 1988 é um direito social, o órgão regulador é a ANVISA. Para Rosenfeld e Costa (2000, p. 17), a instrumentalização legal acontece pela natureza jurídico-política da intervenção praticada pela Vigilância Sanitária, disciplinando aspectos técnico-sanitários da vida em sociedade. Essa instrumentalização atinge a sociedade com um caráter interdisciplinar, agindo na prevenção, através dos atos normativos e na fiscalização dos produtos, serviços e estabelecimentos de saúde.

Com abordagem funcionalista, prescritiva, coercitiva e critérios quantitativos o documento que auxilia o arquiteto no pré-dimensionamento, programa e infraestrutura é a resolução RDC 50, que atualmente está em processo de 
revisão. Outras resoluções atuam em especialidades distintas como Centrais de Material Esterilizado, Endoscopia, Serviços de Diálise, para citar.

Figura 2- Planta Humanizada

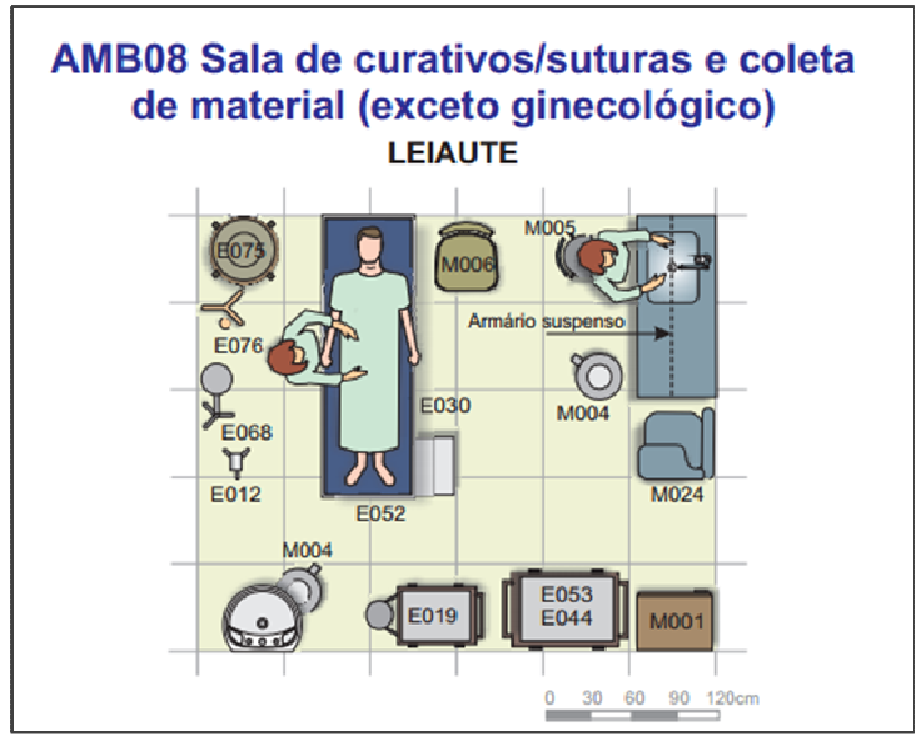

Fonte: SOMASUS

Planta humanizada do programa SOMASUS com pré-dimensionamento e locação de equipamentos

Ainda segundo Rosenfeld e Costa (2000, p. 17), "Para que a Vigilância Sanitária possa cumprir suas finalidades [...] suas práticas devem articular-se, integrar-se, estrategicamente ao conjunto das práticas sanitárias, com o uso de vários instrumentos, com participação e controle social. "

O Ministério da Saúde implementou conceitos atuais de assistência da saúde, no projeto pioneiro Humaniza SUS, que é apoiada em três princípios: inseparabilidade entre a atenção e a gestão dos processos de produção de saúde, transversalidade e autonomia e protagonismo dos sujeitos. É digno refletir que tais conceitos já estão inseridos no pensamento coletivo demostrando a relação sócio-política da saúde.

\section{PROCEDIMENTOS MÉDICOS}

Segundo Focault (2002, p. 109) concomitantemente "[...] o hospital é concebido como um instrumento de cura e a distribuição do espaço torna-se um instrumento terapêutico".

Os procedimentos determinam o espaço usando os princípios arquitetônicos e ergonômicos na análise das áreas necessárias para instalação, utilização de equipamentos, assim como os fluxos de profissionais, pacientes e equipamentos, garantindo um dimensionamento mínimo, ora previsto na normativa ora dependendo do bom senso e do conhecimento adquirido pelo profissional.

Conhecer os processos é o diferencial do arquiteto de saúde, o profissional alia esse conhecimento às necessidades explicitas e implícitas do espaço e dos usuários, cabendo o auxílio das experiências acumuladas por todos agentes do processo. 
Para Malkin (2008, p. 80), as experiências podem ser desenhadas auxiliando a interface do paciente e o processo. Essa técnica está inserida em um estudo maior, o Evidence-Based Design (Desenho Baseado em Evidências) que através dessas experiências e da criação de um banco de dados orienta o processo projetual e o torna mais completo e específico.

Assim, em um ambiente onde procedimentos são rotineiros, a padronização do espaço reduz consideravelmente a ocorrência de erros médicos. Um profissional que manuseia uma medicação (ou equipamento) sempre localizado a sua direita tem maior possibilidade de erro se a medicação estiver à esquerda, dessa forma é recomendável que o espaço destinado a esse procedimento possibilite sempre o manuseio pela direita. Situações como essa nem sempre são previstas por manuais, normas ou tabulações, mas devem fazer parte do repertório do arquiteto, pois influenciam diretamente a segurança do paciente.

\section{DISCUSSÃO}

O processo projetual demanda investigação, maturação e percepção, o planejamento para o espaço mutável requer maior nível de associações com as interferências para prever as transformações futuras. A qualificação do profissional é um item relevante a ser considerado nesse processo sendo caracterizada pelo acúmulo de experiências, a produção de estudos na área e a atualização nos temas.

Nas discussões realizadas por profissionais atuantes no mercado é recorrente o destaque de três elementos que influenciam diretamente o espaço nos ambientes de saúde: a legislação (que previne, fiscaliza, fornece o apoio legal e pré-dimensiona o espaço), o conhecimento das condutas e procedimentos médicos (avaliando e entendendo suas práticas e suas necessidades) e a tecnologia (essencial para infraestrutura, terapia e diagnóstico); essa tríade dialoga, define os fluxos, a compartimentação e setorização conferindo eficiência ao projeto.

Espaços tão complexos e flexíveis com tantas variáveis não é algo solucionável facilmente, o mapeamento dessas determinantes segue como auxilio para os pensamentos preliminares e as intervenções em ambientes de saúde. Por isso, torna-se pertinente repensar e avaliar o processo de projeto dominando tais elementos, assim contribuindo significativamente para a eficácia do resultado.

\section{CONSIDERAÇÕES FINAIS}

Os edifícios de saúde sofrem uma evolução constante e acelerada é imprescindível uma coordenação atenta, a falta de planejamento e as medidas paliativas em nada contribuem com a funcionalidade do espaço. A excelência é conquistada com uma arquitetura interdisciplinar, preditiva e inclusiva.

O campo de conhecimento do arquiteto de instituições de saúde é, assim como o projeto, mutável. A reciclagem do profissional é contínua, as experiências e a atenção aos usuários uma fonte inesgotável de aprendizado. Os profissionais de projeto, administração, tecnologia e o corpo médico precisam interagir com um objetivo em comum: a qualidade do ambiente de 
saúde. Não podemos prever o futuro, mas planejar e direcionar as modificações no espaço é sim algo possível.

\section{AGRADECIMENTOS}

Ao Espaço Saúde (Proarq) da Faculdade de Arquitetura e Urbanismo - UFRJ, pelo curso de Especialização em Ambientes de Saúde (2014).

\section{REFERÊNCIAS}

BRASIL. ANVISA. RDC 50/2002. Regulamento Técnico destinado ao planejamento, programação, elaboração, avaliação e aprovação de projetos físicos de estabelecimentos assistenciais de saúde. Disponível em: <http://portal.anvisa.gov.br/wps/wcm/connect/ca36b200474597459fc8df3fbc 4c6735/RDC+N\%C2\%BA.+50,+DE+21+DE+FEVEREIRO+DE+2002.pdf?MOD=AJPER ES>. Acesso em: 15 jan. 2015. Acesso em: 30 mar. 2015.

BRASIL, Ministério da Saúde, Secretaria Executiva. Departamento de Economia da saúde e Desenvolvimento. SOMA SUS, Programação Arquitetônica de Unidades Funcionais de Saúde Atendimento Ambulatorial e Atendimento Imediato. Brasília: MS, 2011.

FOCAULT, M. Microfísica do poder. 17ed. Rio de Janeiro: Edições Gaal, 2002, p. 109.

KARMAN, J. Manutenção e segurança hospitalar preditivas. 2ed. São Paulo: Estação Liberdade: IPH, 2011 , p. 31.

MACHRY, H. S. O impacto dos avanços da tecnologia nas transformações arquitetônicas dos edifícios hospitalares. Anais do V Congresso Brasileiro para o desenvolvimento do edifício hospitalar, São Paulo, 2012, p. 82.

MALKIN, J. A Visual Reference for Evidence-Based Design. Concord: The Center for Health Design, 2008, p. 80.

ROZENFELD, S.; COSTA, E. A. Constituição da Vigilância Sanitária no Brasil, Fundamentos da Vigilância Sanitária. Rio de Janeiro: FIOCRUZ, 2000, p. 15-40. 